\title{
EPIDEMIOLOGÍA Y CONTROL DE LA CISTICERCOSIS EN EL PERÚ
}

\author{
Hector H. Garcia ${ }^{1,2,3, a, c}$, Armando E. Gonzalez ${ }^{4, d}$, Silvia Rodriguez ${ }^{3, e, f}$, Guillermo Gonzalvez $2, a$, \\ Fernando Llanos-Zavalagaa, ${ }^{5, a, b}$, Víctor C.W. Tsang ${ }^{6, c, g}$, Robert H. Gilman ${ }^{7, a, h}$, \\ por el Grupo de Trabajo en Cisticercosis en Perú
}

\begin{abstract}
RESUMEN
La neurocisticercosis, infección del sistema nervioso humano por el estadio larvario de la Taenia solium, es una causa importante de epilepsia y otras manifestaciones neurológicas en el Perú y en la mayoría de países en desarrollo. Desde 1987, el Grupo de Trabajo en Cisticercosis en Perú ha desarrollado una serie de estudios epidemiológicos que han llevado a estimar el impacto y entender la transmisión de la Taenia solium, y que posteriormente se aplicaron al diseño y ejecución de un programa de control en Tumbes, en la costa norte del país. En este artículo se revisan los principales hallazgos epidemiológicos, así como las líneas generales del programa de eliminación y las herramientas utilizadas. Los avances en el control de la teniasis/cisticercosis en nuestro país abren el camino hacia su eliminación y eventual erradicación.
\end{abstract}

Palabras clave: Cisticercosis; Taenia solium; Epidemiología; Control de enfermedades transmisibles; Perú (fuente: DeCS BIREME).

\section{EPIDEMIOLOGY AND CONTROL OF CYSTICERCOSIS IN PERU}

\begin{abstract}
Neurocysticercosis, the infection of the human central nervous system by the larval stage of the cestode Taenia solium, is an important cause of epilepsy and other neurological manifestations in Peru and most developing countries. Since 1987, the Cysticercosis Working Group in Peru has performed a series of epidemiological studies which led to estimate the impact and to better understand the transmission of Taenia solium. This information was later applied to the design and execution of a control program in Tumbes, in the Northern Coast of Peru. This paper reviews the main epidemiological findings, as well as the conceptual framework of the elimination program and the tools used. Advances in the control of taeniasis/cysticercosis in our country open the road towards its elimination and potential eradication.
\end{abstract}

Key words: Cysticercosis; Taenia solium; Epidemiology; Communicable disease control; Peru (source MeSH NLM).

\section{INTRODUCCIÓN}

La neurocisticercosis es un contributor importante a la patología neurológica en el Perú. Los primeros reportes locales datan de 1915 (1), y existe una abundante cantidad de literatura nacional sobre el tema. A diferencia de las descripciones clínicas, sus características epidemiológicas son mucho menos entendidas y escasamente reportadas hasta inicios de los años 90, principalmente por la falta de herramientas con la suficiente sensibilidad y especificidad para permitir análisis y conclusiones válidas. El Grupo de Trabajo de Cisticercosis en Perú fue uno de los primeros grupos de investigación en el mundo, en aplicar la técnica de inmunoblot en el estudio epidemiológico de la cisticercosis, a lo que luego agregaría estudios poblacionales imagenológicos y de carga de enfermedad. Basados en esta información, se pasó a continuación, a la prueba de intervenciones de control con miras a la eliminación de la transmisión.

La Taenia solium es un cestodo o gusano plano segmentado. El adulto vive solamente en el intestino delgadohumanoy excretasushuevos con las deposiciones del individuo infectado, libres o dentro de segmentos del gusano (proglótidos). El estadio larvario o cisticerco es

\footnotetext{
Departamento de Microbiología, Universidad Peruana Cayetano Heredia, Lima, Perú.

2 Centro de Salud Global - Tumbes, Universidad Peruana Cayetano Heredia, Tumbes, Perú.

Unidad de Cisticercosis, Departamento de Neuropediatría y Enfermedades Transmisibles, Instituto Nacional de Ciencias Neurológicas, Lima, Perú.

Facultad de Medicina Veterinaria, Universidad Nacional Mayor de San Marcos, Lima, Perú.

Facultad de Salud Pública y Administración, Universidad Peruana Cayetano Heredia, Lima, Perú.

Georgia State University, Atlanta, Georgia, USA.

Departamento de Salud Internacional, Universidad Johns Hopkins, Baltimore, Maryland, USA.

Médico; ' ${ }^{b}$ Magíster en Salud Pública; ' Doctor en Salud Internacional; ${ }^{\mathrm{d}}$ Médico Veterinario, Doctor en Epidemiología Veterinaria; ${ }^{\mathrm{e}}$ Biólogo; ${ }^{\mathrm{f}}$ Magíster

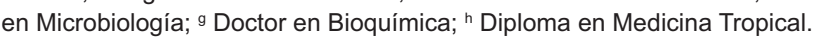

Recibido: 14-11-10 Aprobado: 01-12-10 
una vesícula blanquecina de aproximadamente $1 \mathrm{~cm}$, con contenido líquido, que tiene en su parte interna la cabeza o escólex a partir de la cual crece el gusano adulto (2). El hospedero intermediario usual es el cerdo, que se infecta ingiriendo heces humanas contaminadas con huevos o proglótidos de $T$. solium, merced a la coexistencia de crianza doméstica de cerdos y pobre saneamiento. El humano se infecta por contaminación fecal, y la infección es más frecuente en el entorno inmediato del portador de la tenia adulta ${ }^{(2,3)}$.

La teniasis o infección por el gusano adulto, intestinal, cursa asintomática o produce síntomas digestivos leves e inespecíficos. En cambio, la presencia de quistes en el sistema nervioso o en el globo ocular esta frecuentemente asociada con sintomatología. La neurocisticercosis (NCC) se puede presentar como casi cualquier síntoma neurológico, particularmente crisis epilépticas, de inicio luego de la segunda década de la vida, y la cisticercosis ocular causa alteraciones de la visión. La cisticercosis fuera del sistema nervioso o del ojo es usualmente asintomática, pudiéndose notar ocasionalmente las larvas en el tejido celular subcutáneo ${ }^{(4)}$.

La teniasis se diagnostica principalmente con exámenes de heces. El método clásico de examen microscópico

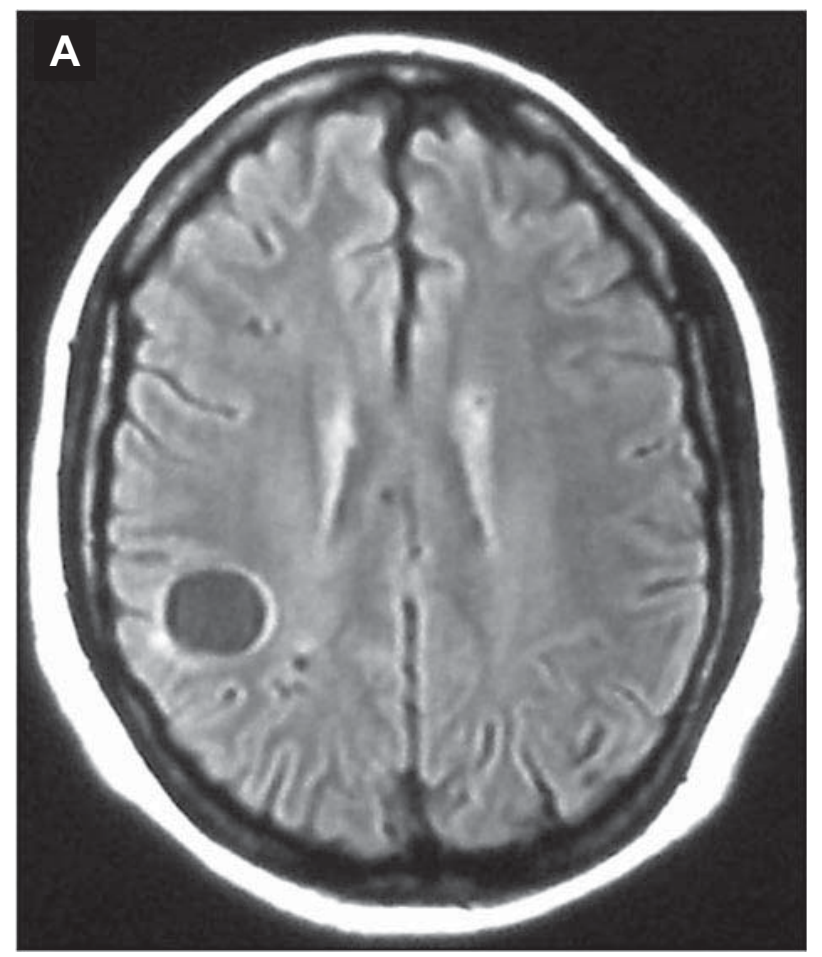

luego de sedimentar la muestra tiene alta especificidad, pero desafortunadamente baja sensibilidad ${ }^{(5)}$. Existe una prueba de ELISA para detectar antígenos parasitarios en heces (coproantígenos), que tiene mucha mas sensibilidad pero cuyo uso es aún restringido al contexto experimental y no se encuentra en los centros asistenciales ${ }^{(6)}$. La neurocisticercosis se diagnostica por exámenes de imagen (tomografía computarizada o resonancia magnética) (Figura 1), confirmándose por serología con la técnica de western blot ${ }^{(7)}$.

El tratamiento de la teniasis utiliza dosis únicas orales de niclosamida ( $2 \mathrm{~g}$ en adultos, $1 \mathrm{~g}$ en niños) o praziquantel $(5 \text { a } 10 \mathrm{mg} / \mathrm{kg})^{(8,9)}$. El tratamiento de la neurocisticercosis en general involucra tanto manejo sintomático como antiparasitario. El manejo sintomático tiene por objeto controlar las manifestaciones clínicas, particularmente las crisis epilépticas o solucionar la presencia de hipertensión endocraneal. El tratamiento antiparasitario depende del estado, número, tamaño y localización de los quistes, y se usa albendazol $(15 \mathrm{mg} / \mathrm{k} / \mathrm{d}$ por una a dos semanas) o praziquantel ( $50 \mathrm{mg} / \mathrm{kg} / \mathrm{d}$ por dos semanas), usualmente con esteroides. Es importante individualizar el manejo de cada paciente por que hay situaciones donde el tratamiento antiparasitario puede estar contraindicado ${ }^{(4)}$.

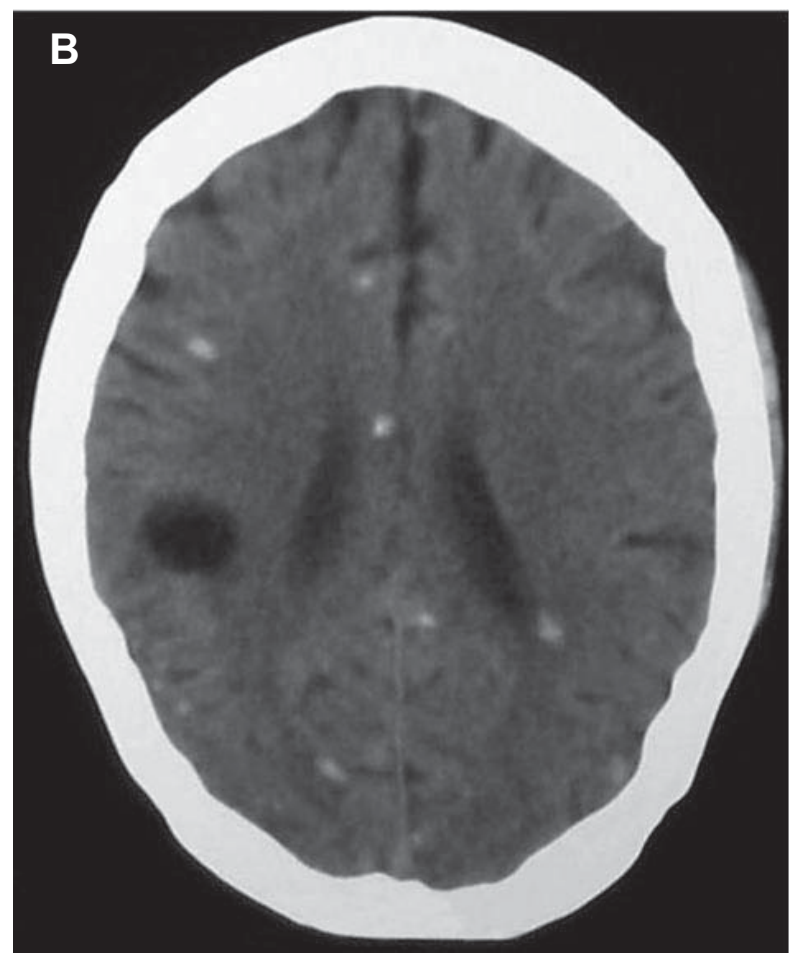

Figura 1. Resonancia magnética (a) y tomografía computarizada (b) cerebrales mostrando los diferentes estadios de la cisticercosis. 


\section{EPIDEMIOLOGÍA DE LA TENIASIS/ CISTICERCOSIS EN EL PERÚ}

Entre 1990 y 1995, a partir de la introducción de la técnica serológica de western blot, se llevó a cabo una serie de estudios seroepidemiológicos en grupos poblacionales definidos, demostrándose seroprevalencias basales de entre 10 y $20 \%$ en población general, con valores dos a tres veces más altos en individuos con epilepsia, de las mismas regiones. Esta información confirmó el rol de la neurocisticercosis en la etiología de la epilepsia en zonas endémicas ${ }^{(10-12)}$. Basados en la procedencia de los casos clínicos y los estudios de prevalencia de nuestro grupo, estimamos como las zonas más claramente endémicas para NCC toda la sierra, la costa norte y la selva alta (Figura 2). Interesantemente, tanto la costa norte como la selva alta son focos receptores de migración de zonas endémicas de la sierra. La NCC está presente en menores cantidades en el resto del país, con lquitos aparentemente libre de enfermedad, probablemente por su aislamiento del resto de departamentos. La teniasis se encuentra en promedio entre 0,5 a $1 \%$ de pobladores en áreas endémicas, con mayor prevalencia en jóvenes, mujeres embarazadas y ancianos. Hay pequeñas

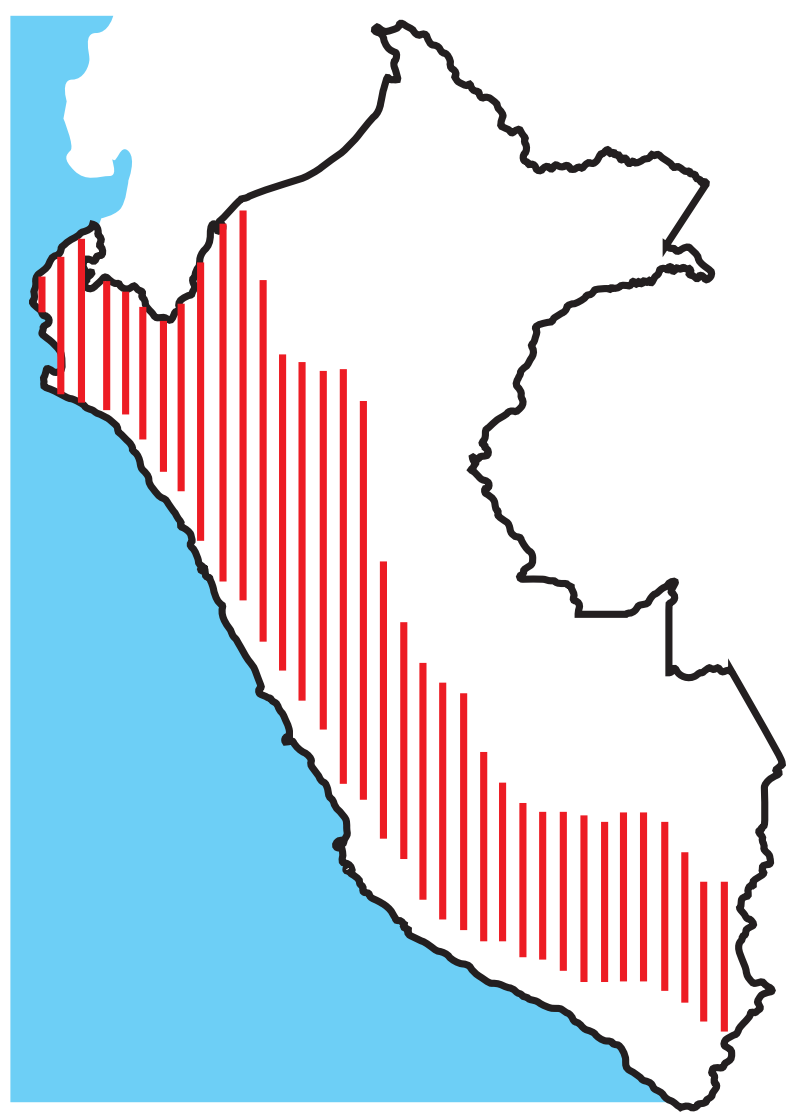

Figura 2. Mapa del Perú mostrando las principales áreas endémicas para teniasis/cisticercosis por Taenia solium diferencias genéticas entre tenias colectadas en el norte y el sur del país.

De otro lado, existen muy pocos datos sobre prevalencia o incidencia de epilepsia en el Perú. Los datos sobre prevalencia (aproximadamente 15/1000) e incidencia (entre 90 y 150/100 000) en Tumbes son dos a tres veces más altos que los correspondientes valores en USA y Europa. Los estudios serológicos o de imagen en personas con epilepsia en zonas endémicas, encuentran consistentemente entre 30 y $50 \%$ de casos asociados con neurocisticercosis ${ }^{(13,14)}$. Bern et al. publicaron en 1999 un análisis que estima en 400000 el número de casos de NCC sintomática en Latinoamérica y 31000 en Perú ${ }^{(15)}$. Rajkoitia et al. estimaron el costo usual en $54 \%$ de un salario mínimo anual ${ }^{(16)}$. Ya en el 2005 , los datos de los estudios en Tumbes llevaron a la conclusión de que la NCC contribuye en un $30 \%$ de los síndromes epilépticos en esta región, dato que es compatible con estudios en Honduras y Ecuador publicados por las mismas fechas $(13,17,18)$.

\section{CONTROL DE LA TENIASIS/CISTICERCOSIS}

Desde inicios de la década de 1980 , se han probado diferentes intervenciones para el control de la teniasis/ cisticercosis, en adición a las medidas estándar de control en camales. Los estudios iniciales utilizaron tratamiento masivo de la población humana con praziquantel o niclosamida y educación en salud, a lo que en años recientes se ha agregado tratamiento de la población porcina con oxfendazole y vacunación de la población porcina. Estas medidas se analizan en detalle a continuación.

Control en camales. Clásicamente recomendado, el control en camales ha fallado claramente en controlar la transmisión. Las razones por las que falla involucran inadecuada legislación (los campesinos están sujetos a la confiscación del animal infectado sin ningún tipo de retribución, lo que hace que llevar los animales al camal sea un riesgo), falta de seguimiento de los focos de infección, y la limitada cantidad de cortes que se pueden hacer a la carcasa para no estropearla (en el marco de una infección donde la mayoría de animales infectados tienen menos de diez quistes en total) ${ }^{(19,20)}$.

Tratamiento masivo de la población humana con praziquantel o niclosamida. Inicialmente aplicada en el sur del Ecuador, la quimioterapia masiva con praziquantel mostró ser temporalmente eficaz en disminuir la prevalencia de teniasis y de cisticercosis porcina (21). Subsecuentemente, esto se replicó en México, Guatemala, Perú y otros países, con similares resultados ${ }^{(22-25)}$. La 
información disponible sugiere que el efecto es temporal y la prevalencia regresa rápidamente a sus valores basales en meses o años luego de la suspensión de las campañas de desparasitación. Existe también la duda teórica sobre si los tratamientos masivos con praziquantel (droga aprobada y eficaz contra la teniasis, pero que se absorbe a sangre) podrían desencadenar crisis epilépticas en portadores asintomáticos de cisticercosis cerebral. La otra alternativa terapéutica es niclosamida, que no presenta este potencial riesgo por que no se absorbe. Tanto praziquantel como niclosamida son de pobre disponibilidad en la mayoría de países.

Educación en salud. Componenteobligado de cualquier programa de salud pública, la educación sanitaria ha sido empleada en el control de la cisticercosis con resultados favorables en México. Enfoques más recientes apuntan a orientar la educación hacia el logro de objetivos específicos, como lavado de manos, uso de letrinas, etc ${ }^{(26)}$

Tratamiento de la población porcina con oxfendazole. Nuestro grupo demostró en 1996 la eficacia del oxfendazole para curar la cisticercosis porcina en una sola dosis de $30 \mathrm{mg} / \mathrm{kg}$. Su aplicación al control debe resultar en una disminución significativa del tiempo necesario para lograr el control al eliminar la población de larvas y con ella las posibilidades de nuevos casos de teniasis. Un primer estudio de eficacia fue luego complementado con un estudio de precisión de dosis efectiva y otro de estimación del tiempo de sobrevida de los quistes luego del tratamiento (estimado en un mes) (27-29).

Vacunación de la población porcina. El grupo de parasitología veterinaria de la Universidad de Melbourne, Australia, encabezado por el Dr. Marshall Lightowlers, desarrolló una vacuna altamente eficaz para la cisticercosis porcina, probada inicialmente en México, Camerún y Perú a inicios de la primera década del 2000. Esta vacuna, llamada TSOL18, tiene una eficacia superior al $99 \%$ y fue desarrollada siguiendo el esquema de vacunas similares contra Taenia ovis y Taenia saginata ${ }^{(30,31)}$.

\section{EL PROGRAMA DE ELIMINACIÓN DE LA CISTICERCOSIS EN TUMBES, PERÚ}

La cisticercosis ha sido conceptualizada como una de las pocas enfermedades erradicables, es decir potencialmente capaces de ser desaparecidas de la faz de la tierra. Las razones por las cuales se considera erradicable incluyen el hecho de tener un solo hospedero definitivo, tener al cerdo que es un animal doméstico y de fácil accesibilidad como hospedero intermediario usual, y la existencia de tratamientos efectivos para el gusano adulto o la fase larvaria ${ }^{(32,33)}$. Después de muchos años de trabajar en la epidemiología de esta enfermedad y luego de algunas experiencias iniciales en control, el Grupo de trabajo en Cisticercosis en Perú decidió acometer la tarea de demostrar la factibilidad de la eliminación de la transmisión de la cisticercosis, bajo el apoyo de un proyecto de control de siete años financiado por la Fundación Bill \& Melinda Gates de los EE.UU.

El programa partió de las siguientes bases conceptuales: a) Taenia solium tiene un enorme potencial biótico (capacidad de reproducción) y b) La teniasis/cisticercosis es una enfermedad asociada con la pobreza y dentro de las enfermedades más desatendidas. Las implicancias de estos conceptos llevan al diseño de un programa de intensa intervención y prolongada vigilancia, ya que unos pocos gusanos que sobrevivan la intervención podrían reinstalar la endemicidad de la enfermedad. Al mismo tiempo, el costo de la intervención debe ser bajo y estar en capacidad de aplicarse a través de las estructuras locales de salud y agricultura, probablemente bajo subvención estatal. Es difícil pensar que los campesinos van a invertir en el control de la cisticercosis en sus cerdos si no invierten en enfermedades de alta mortandad como el cólera porcino ${ }^{(34)}$.

Se utilizaron varias medidas en simultáneo: tratamiento de la población humana con niclosamida con identificación de casos vía detección de coproantígeno, tratamiento de la población porcina con niclosamida y vacunación de la población porcina. Se seleccionó la niclosamida antes que el praziquantel en base a los datos publicados de alta eficacia (superior al 95\%), y por que no conlleva la posibilidad teórica de ocasionar síntomas neurológicos en individuos que pudieran tener NCC latente. Sin embargo, los datos de seguimiento postratamiento mostraron que la eficacia de la niclosamida en nuestro programa fue bastante más baja de lo esperado, con valores de alrededor del $65 \%$. La niclosamida se dio en campañas de tratamiento masivo, con dosis estándar y teniendo cuidado de moler finamente las pastillas antes de su administración. A pesar del gran número de tratamientos administrados, los efectos adversos fueron mínimos y pasajeros, sin ningún evento adverso grave. Se solicitó una muestra de heces postratamiento a cada persona tratada. Estas muestras fueron procesadas por ELISA para detección de coproantígenos, y los casos positivos fueron seguidos con muestras a las dos y cuatro semanas postratamiento para confirmar la cura o identificar las fallas de tratamiento, quienes recibieron un segundo tratamiento dirigido, previa aplicación de un purgativo. 


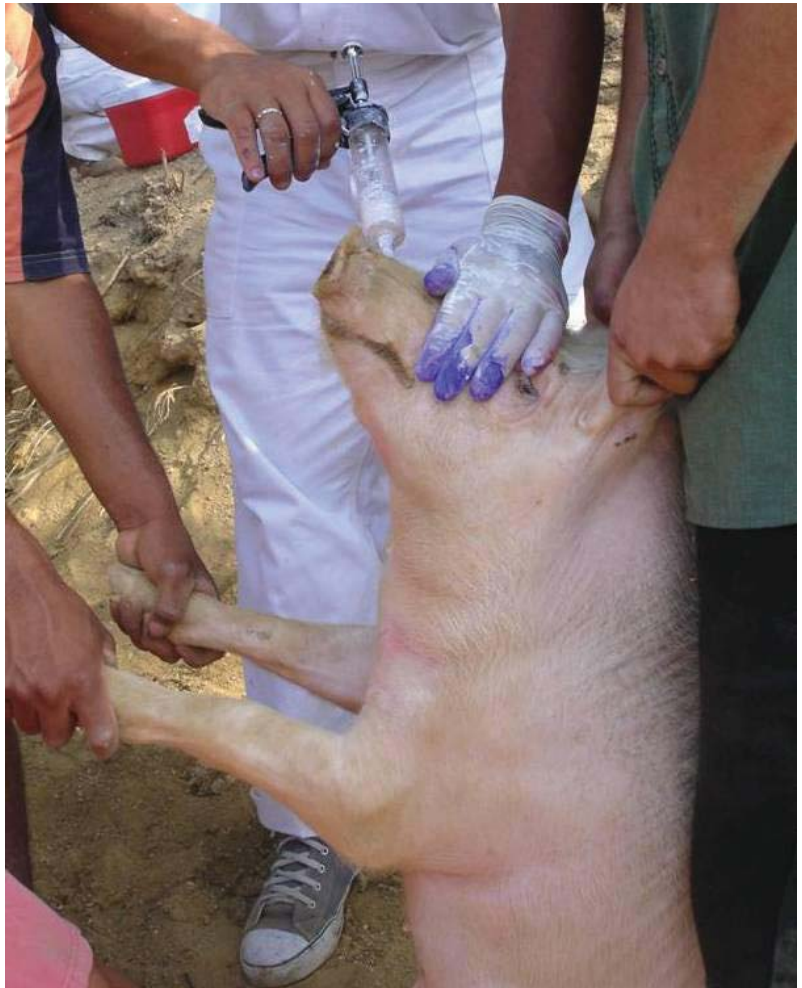

Figura 3. Administración de oxfendazole a un cerdo en el marco de un programa de control en el campo.

En la población porcina se usaron dosis orales de 30 $\mathrm{mg} / \mathrm{kg}$ de oxfendazole. Esta droga se administró a todos los cerdos mayores de dos meses, presentes en las comunidades al tiempo de cada ronda de intervención (Figura 3). Adicionalmente, en colaboración con la Universidad de Melbourne en Australia, pudimos contar con la efectiva vacuna TSOL18 para la cisticercosis porcina. Esta vacuna, de eficacia cercana al $100 \%$, se administró a todos los cerdos mayores de dos años, en dos dosis separadas por dos semanas.

Inicialmente, se seleccionó la incidencia serológica (nuevos casos de seroconversión en cerdos previamente seronegativos) como indicador principal de eficacia del programa de control. Sin embargo, el elevado nivel basal de seroprevalencia y el hecho de que muchos animales seroconvierten pero no desarrollan infección, nos llevaron a utilizar en la segunda ronda del programa una combinación de tamizaje serológico con necropsia detallada de los animales seropositivos buscando quistes viables, como gold standard de infección y, como tal, evidencia de transmisión activa.

Luego de dos rondas iniciales de evaluación de eficacia, se aplicó la intervención a toda el área rural de la región Tumbes. Al final del año de intervención, la evaluación sistemática de porcinos con necropsia detallada demostró que no había huellas de transmisión en el $95 \%$ de las comunidades intervenidas. Luego de esta prueba de factibilidad, la siguiente etapa del control implica el desarrollo de sistemas de vigilancia para evitar la reintroducción, la optimización de las herramientas en términos de costo y disponibilidad y la expansión del área de eliminación.

El camino de la eliminación de la teniasis/cisticercosis por Taenia solium está en sus inicios. Las evidencias obtenidas apuntan a que este objetivo es factible y, en los años siguientes, debemos entrar a una etapa de operacionalización de las intervenciones en versiones más simples y potencialmente aplicables en otras realidades. Una vez definido un esquema factible y de costos alcanzables con una estrategia eficaz de vigilancia (que probablemente involucre declarar la teniasis enfermedad de notificación obligatoria en las zonas de eliminación), estos deberán entonces ser insertados en los sistemas regionales de salud y agricultura para su expansión.

\section{Conflictos de Interés}

Los autores declaran no tener conflictos de interés en la publicación de este artículo.

\section{REFERENCIAS BIBLIOGRÁFICAS}

1. Hercelles $\mathrm{O}$, Voto-Bernales J. La cisticercosis humana en el Peru. Cronica Med. 1915; 621: 49-54.

2. Flisser A. Taeniasis and cysticercosis due to Taenia solium. Prog Clin Parasitol. 1994; 4: 77-116.

3. Nash TE, Neva FA. Recent advances in the diagnosis and treatment of cerebral cysticercosis. $\mathrm{N}$ Engl $\mathrm{J}$ Med. 1984;311(23): 1492-6.

4. Garcia HH, Del Brutto OH. Neurocysticercosis: updated concepts about an old disease. Lancet Neurol. 2005;4(10):653-61.

5. Mayta H, Talley A, Gilman RH, Jimenez J, Verastegui M, Ruiz M, et al. Differentiating Taenia solium and Taenia saginata infections by simple hematoxylin-eosin staining and PCR-restriction enzyme analysis. J Clin Microbiol. 2000;38(1):133-7.

6. Allan JC, Avila G, Garcia Noval J, Flisser A, Craig PS. Immunodiagnosis of taeniasis by coproantigen detection. Parasitology. 1990;101(Pt 3):473-77.

7. Del Brutto OH, Rajshekhar V, White AC, Jr., Tsang VC, Nash TE, Takayanagui OM, et al. Proposed diagnostic criteria for neurocysticercosis. Neurology. 2001;57(2):177-83.

8. Pearson RD, Guerrant RL. Praziquantel: a major advance in anthelminthic therapy. Ann Intern Med. 1983;99(2):195-98.

9. Pearson RD, Hewlett EL. Niclosamide therapy for tapeworm infections. Ann Intern Med. 1985;102(4):550-51.

10. Diaz F, Garcia HH, Gilman RH, Gonzales AE, Castro M, Tsang VC, et al. Epidemiology of taeniasis and cysticercosis in a Peruvian village. The Cysticercosis Working Group in Peru. Am J Epidemiol. 1992;135(8):875-82. 
11. Garcia HH, Araoz R, Gilman RH, Valdez J, Gonzalez AE, Gavidia C, et al. Increased prevalence of cysticercosis and taeniasis among professional fried pork vendors and the general population of a village in the Peruvian highlands. Cysticercosis Working Group in Peru. Am J Trop Med Hyg. 1998;59(6):902-5.

12. Garcia HH, Gilman R, Martinez M, Tsang VC, Pilcher JB, Herrera G, et al. Cysticercosis as a major cause of epilepsy in Peru. The Cysticercosis Working Group in Peru (CWG). Lancet. 1993;341(8839):197-200.

13. Montano SM, Villaran MV, Ylquimiche L, Figueroa JJ, Rodriguez S, Bautista $\mathbf{C T}$, et al. Neurocysticercosis: association between seizures, serology, and brain $\mathrm{CT}$ in rural Peru. Neurology. 2005; 65(2): 229-33.

14. Villaran MV, Montano SM, Gonzalvez G, Moyano LM, Chero JC, Rodriguez S, et al. Epilepsy and neurocysticercosis: an incidence study in a Peruvian rural population. Neuroepidemiology. 2009; 33(1): 25-31.

15. Bern C, Garcia HH, Evans C, Gonzalez AE, Verastegui M, Tsang VC, et al. Magnitude of the disease burden from neurocysticercosis in a developing country. Clin Infect Dis. 1999; 29(5): 1203-9.

16. Rajkotia Y, Lescano AG, Gilman RH, Cornejo C, Garcia HH. Economic burden of neurocysticercosis: results from Peru. Trans R Soc Trop Med Hyg. 2007; 101(8): 840-6.

17. Del Brutto $\mathrm{OH}$, Santibanez R, Idrovo L, Rodriguez S, DiazCalderon E, Navas C, et al. Epilepsy and neurocysticercosis in Atahualpa: a door-to-door survey in rural coastal Ecuador. Epilepsia. 2005; 46(4): 583-7.

18. Medina MT, Duron RM, Martinez L, Osorio JR, Estrada AL, Zuniga C, et al. Prevalence, incidence, and etiology of epilepsies in rural Honduras: the Salama Study. Epilepsia. 2005; 46(1): 124-31.

19. Gemmell M, Matyas Z, Pawlowsky Z, Soulsby EJL. Guidelines for surveillance and control of taeniasis/ cysticercosis. Geneva: World Health Organization; 1983.

20. The Cysticercosis Working Group in Peru. The marketing of cysticercotic pigs in the Sierra of Peru. Bull World Health Organ. 1993;71(2):223-28

21. Cruz M, Davis A, Dixon H, Pawlowski ZS, Proano J. Operational studies on the control of Taenia solium taeniasis/cysticercosis in Ecuador. Bull World Health Organ. 1989;67(4):401-7.

22. Sarti E, Schantz PM, Avila G, Ambrosio J, Medina-Santillan R, Flisser A. Mass treatment against human taeniasis for the control of cysticercosis: a population-based intervention study. Trans R Soc Trop Med Hyg. 2000;94(1):85-89.

23. Keilbach NM, de Aluja AS, Sarti-Gutierrez E. A programme to control taeniasis-cysticercosis ( $T$. solium): experiences in a Mexican village. Acta Leiden. 1989;57(2):181-89.
24. Allan JC, Velasquez-Tohom M, Fletes C, Torres-Alvarez R, Lopez-Virula G, Yurrita P, et al. Mass chemotherapy for intestinal Taenia solium infection: effect on prevalence in humans and pigs. Trans R Soc Trop Med Hyg. 1997;91(5):59598.

25. Diaz Camacho SP, Candil Ruiz A, Suate Peraza V, Zazueta Ramos ML, Felix Medina M, Lozano R, et al. Epidemiologic study and control of Taenia solium infections with praziquantel in a rural village of Mexico. Am J Trop Med Hyg. 1991;45(4):522-31

26. Sarti E, Flisser A, Schantz PM, Gleizer M, Loya M, Plancarte $\mathbf{A}$, et al. Development and evaluation of a health education intervention against Taenia solium in a rural community in Mexico. Am J Trop Med Hyg. 1997;56(2):12732.

27. Gonzales AE, Garcia HH, Gilman RH, Gavidia CM, Tsang VC, Bernal T, et al. Effective, single-dose treatment or porcine cysticercosis with oxfendazole. Am J Trop Med Hyg. 1996;54(4):391-94

28. Gonzalez AE, Falcon N, Gavidia C, Garcia HH, Tsang VC, Bernal T, et al. Treatment of porcine cysticercosis with oxfendazole: a dose-response trial. Vet Rec. 1997;141(16):420-22.

29. Gonzalez AE, Falcon N, Gavidia C, Garcia HH, Tsang VC, Bernal T, et al. Time-response curve of oxfendazole in the treatment of swine cysticercosis. Am J Trop Med Hyg. 1998;59(5):832-36.

30. Gonzalez AE, Gauci CG, Barber D, Gilman RH, Tsang VC, Garcia HH, et al. Vaccination of pigs to control human neurocysticercosis. Am J Trop Med Hyg. 2005;72(6):837-39.

31. Flisser A, Gauci CG, Zoli A, Martinez-Ocana J, GarzaRodriguez A, Dominguez-Alpizar JL, et al. Induction of protection against porcine cysticercosis by vaccination with recombinant oncosphere antigens. Infect Immun. 2004;72(9):5292-97.

32. Schantz PM, Cruz M, Sarti E, Pawlowski Z. Potential eradicability of taeniasis and cysticercosis. Bull Pan Am Health Organ. 1993;27(4):397-403.

33. Pawlowski ZS. Potential eradication of taeniasis/ cysticercosis. Parasitol Today. 1993;9(12):464.

34. Gonzalez AE, Garcia HH, Gilman RH, Tsang VCW, The Cysticercosis Working Group in Peru. Control of Taenia solium. Acta Tropica. 2003;87(1):103-9.

Correspondencia: Hector H. Garcia.

Departamento de Microbiología UPCH, Av. H. Delgado 430, SMP, Lima 31, Perú.

Teléfono: 3287360

Correo electrónico: hgarcia@jhsph.edu 